\title{
Improved Simulation of Quasi-Optical Launchers for High Power Gyrotrons with Smoothing Algorithm
}

\author{
Jianbo Jin ${ }^{1}$, Gerd Gantenbein ${ }^{1}$, Tomasz Rzesnicki ${ }^{1}$, Manfred Thumm ${ }^{1,2}$, and John Jelonnek ${ }^{1,2}$ \\ ${ }^{1} \mathrm{IHM},{ }^{2} \mathrm{IHE}$, Karlsruhe Institute of Technology (KIT), Kaiserstr. 12, 76131 Karlsruhe, Germany
}

\begin{abstract}
Numerically optimized launchers for the quasi-optical output coupler of high power gyrotrons have been developed to provide RF beams with high Gaussian mode content. Generally, the profiles of numerically optimized launchers are quite complicated, so the improvement of their tolerance to fabrication errors is very important. In order to reduce the stray radiation generated by launchers with quite complicated wall profile, and also to reduce their sensitivity to fabrication errors, a method for the smoothing of the numerically optimized launcher wall has been developed at KIT, which is based on the spectrum reconstruction approach. As an example, a launcher designed for the KIT TE 34,19 -mode, 2 MW CW coaxialcavity gyrotron has been investigated.
\end{abstract}

\section{Introduction}

Gyrotrons are used as highest-power millimetre wave sources for Electron Cyclotron Resonance Heating (ECRH) of nuclear fusion plasmas. Those megawattlevel gyrotrons are usually operated in very high order cavity modes. Quasi-optical mode converters are employed to transform the short-wavelength and rotating asymmetric high-order cavity mode into a Gaussian-like distribution, which is directly usable for low-loss transmission in free space. The quasi-optical mode converter consists of a specific mode-converting waveguide/launcher and a mirror system. Oversized circular waveguide launchers with step-cut smooth wall were proposed by Vlasov et al. [1,2]. Launchers with harmonically deformed waveguide wall were originally proposed and demonstrated to improve the quality of the RF beams and decrease the diffraction loss of the power radiated from the launcher [3]. In 2006, by use of the Huygens' principle, a numerical method was developed for the synthesis of oversized mirror-line launchers for high power gyrotrons [4]. Later in 2009, a completely different numerical method was developed for the design of such mirror-line launchers at KIT [5]. Base on the integral Lorentz lemma, a systematic approach was proposed for the numerical optimization of multimode waveguides to convert a specific input mode into a different output field [6]. In a next step, a novel numerical method was developed at KIT for the synthesis of hybrid-type launchers [7]. The profiles of the numerically optimized launchers are relatively complicated. In order to smoothen the wall surface to satisfy fabrication requirements and to improve the tolerance of the launcher to fabrication errors and frequency shifts, it is very important to develop a method for smoothing of the surface of the launcher wall in the numerical synthesis procedure. A numerical method for the smoothing of wall surfaces has been developed based on the spectrum reconstruction method [8]. As an example, the mirror-line launcher developed for the KIT $170 \mathrm{GHz} 2 \mathrm{MW} \mathrm{TE}_{34,19}$-mode coaxial-cavity gyrotron has been investigated with and without wall smoothing in terms of the spectrum reconstruction method. In the case of the smoothened launcher wall, the minimum wall contour curvature radius increases from $1.5 \mathrm{~mm}$ (without smoothing) to $29 \mathrm{~mm}$. The simulation results show that the Gaussian mode content of the RF beam at the launcher aperture only decreases from $96.26 \%$ (without smoothing) to $96 \%$. The high power experimental results reveal that the Gaussian mode content of the RF beam is $96 \%$ at the output window, and the stray radiation is decreased from $7 \%$ (without smoothing) to $4 \%$ [9]. From the experimental results one can see that although the Gaussian mode content of the RF beam is slightly decreased when the wall surface is smoothed based on the spectrum reconstruction method, the stray radiation can be obviously reduced. 


\section{Numerically optimized launcher for the $170 \mathrm{GHz}, \mathrm{TE}_{34,19}$-mode coaxial gyrotron}

A mirror-line launcher has been designed for the $\mathrm{TE}_{34,19^{-}}$ mode coaxial gyrotron at KIT [5]. The sketch of the launcher with a helical cut is shown in Fig. 1(a), where $R, \varphi$ and $L$ represent the radius, azimuthal angle and length of the launcher, respectively. In the procedure of the numerical optimization, the wall perturbations are checked in each step to see if the wall profile satisfies the requirement of the minimum curvature radius according to CNC milling machines. In the case that the curvature radius is smaller than the required minimum value, the perturbation of the wall at the sampling point will be modified by linear interpolation according to the neighbour points. If a perturbation value at a sampling point is modified, it will influence the curvature radius of its neighbour points, so the wall perturbations should be iteratively checked to be sure that the curvature radius at each sampling point is larger than the require minimum value. In the numerical synthesis procedure for the mirror-line launcher of the coaxial-cavity gyrotron, the wall perturbations were iteratively checked and modified by linear interpolation, so that the curvature radius at each mesh point should be larger than the minimum allowable curvature radius of $5 \mathrm{~mm}$ according to the tool used to machine the launcher. However, even though the launcher wall was iteratively interpolated 100 times in the synthesis procedure, the curvature radius at a very few mesh points was still as low as $1.8 \mathrm{~mm}$. The wall profile of this non-smoothed mirror-line launcher for the coaxial gyrotron is shown in Fig. 1(b). The curvature radii are calculated via the second derivatives of this profile. Since the gyrotron cavity operates in a TE mode, we use the $\mathrm{H}_{\mathrm{z}}$-field component to show the field distribution in the launcher. The calculated $\mathrm{H}_{\mathrm{z}}$-field distribution on the launcher wall is plotted in Fig. 2. The Gaussian mode content $\eta$ of the RF beam in the launcher is defined as the correlation coefficient of the $\mathrm{H}_{\mathrm{z}}$-field component $u_{z}$ of the wave beam to an ideal Gaussian field distribution $u_{g}$ at the aperture $S$ of the launcher. The edges of the launcher aperture are shown by red lines in Fig. 2, Fig. 6 and Fig. 9.

$$
\eta=\frac{\left|\int_{S} u_{z}^{*} \cdot u_{g} d s\right|^{2}}{\int_{S}\left|u_{z}\right|^{2} d s \cdot \int_{S}\left|u_{g}\right|^{2} d s}
$$

The spectrum of the perturbed wall profile is shown in Fig. 3. As can be seen in Fig. 1(b), the minimum curvature radius in azimuthal direction is estimated as $1.8 \mathrm{~mm}$ and is $10 \mathrm{~mm}$ in longitudinal direction. The radius of the launcher is $32.5 \mathrm{~mm}$.

A mirror system was also designed, manufactured and installed into the coaxial-cavity gyrotron together with the launcher shown in Fig. 1(b). The simulation result shows a Gaussian mode content of the RF beam at the launcher aperture of $96.26 \%$. The high power measurement results reveal that the Gaussian mode content of the output beam is about $96 \%$, and the internal stray radiation in the coaxial-cavity gyrotron is estimated to be $7 \%$. From Fig. 1(b) one can see that there are many "ripples" on the launcher wall, and correspondingly, there are many small-amplitude high-frequency components in the spectrum of the wall profile as shown in Fig. 3. In order to depress the ripples, two methods have been studied to smoothen the perturbed launcher wall.

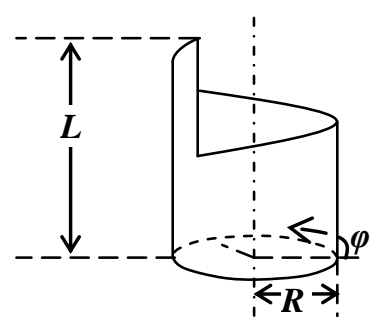

(a)

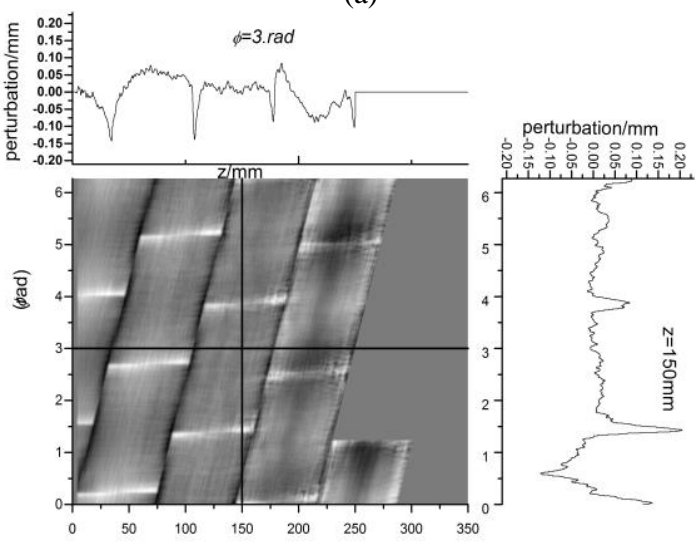

(b)

Fig. 1. (a) Sketch of the launcher, (b) Wall profile of the mirror-line launcher designed for the $170 \mathrm{GHz}$ coaxial-cavity gyrotron.

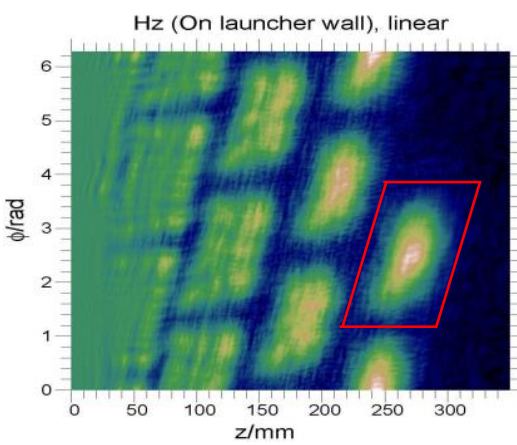

Fig. 2. $\mathrm{H}_{\mathrm{z}}$-field distribution on the wall of the mirror-line launcher (linear scale, $0 \sim 1$ ).

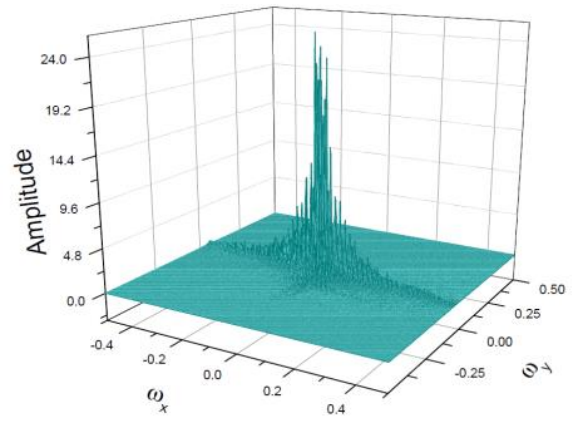

Fig. 3. Spectrum of the non-smoothened launcher wall profile. 


\section{Launcher wall smoothing by low-pass filtering}

The principle of the low-pass filter is shown in Fig. 4. The cut-off frequencies for $\omega_{\mathrm{x}}$ and $\omega_{\mathrm{y}}$ are optimized to keep both the major part of the spectrum shown in Fig. 3 and high Gaussian mode content of the RF beam. The parameters of the low-pass filter for the mirror-line launcher developed for the $\mathrm{TE}_{34,19}$-mode coaxial cavity gyrotron are shown in Fig. 4. In the synthesis procedure, the spectrum of the iteratively interpolated perturbations on the launcher wall was multiplied by the spectrum of the low-pass filter. The filtered spectrum of the profile of the launcher wall is plotted in Fig. 5.

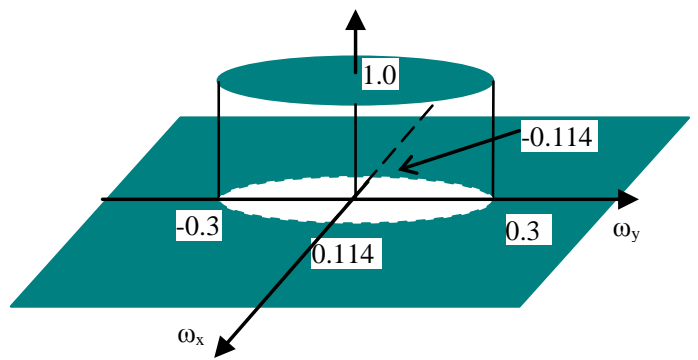

Fig. 4. Low-pass filter for the launcher wall perturbations.

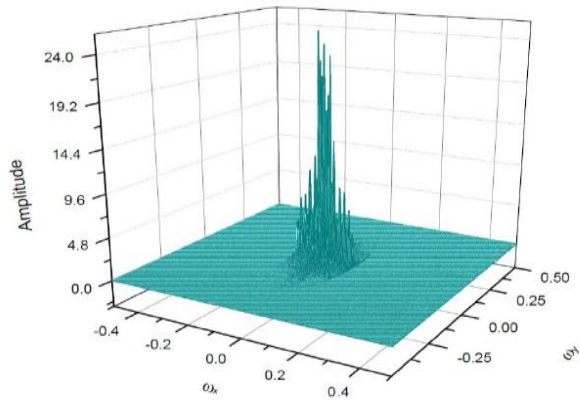

Fig. 5. Spectrum of the filtered launcher wall profile.

The wall profile was generated according to the filtered spectrum in Fig. 5. The simulation results show that the Gaussian mode content of the RF beam is increased a little bit to $96.27 \%$ at the launcher aperture as shown in Fig. 6. The wall profile is similar to that shown in Fig. 1(b), but the minimum curvature radii are increased to $6.4 \mathrm{~mm}$ and $67 \mathrm{~mm}$ in azimuthal direction and longitudinal direction, respectively. Comparing the field distribution shown Fig. 6 with that plotted in Fig. 2, the field contours still look somewhat irregular.

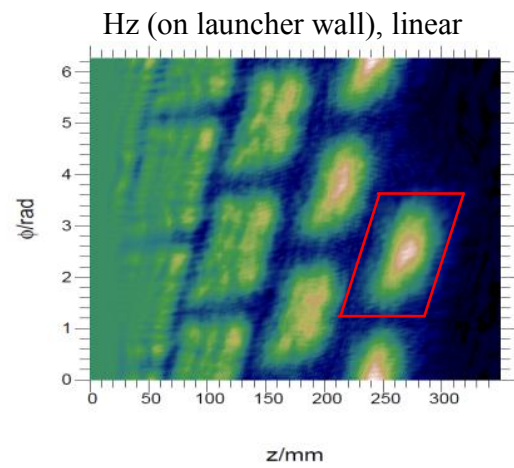

Fig. 6. $\mathrm{H}_{\mathrm{z}}$-field distribution on the wall of the mirror-line launcher smoothed using low-pass filtering (linear scale, $0 \sim 1$ ).

\section{Launcher wall smoothing by the spectrum reconstruction method}

A spectrum reconstruction method has been developed to smoothen the launcher wall furthermore and to increase the minimum curvature radius. The method is described as follows [8]

$F_{N}\left(\omega_{z}, \omega_{\varphi}\right)=\left\{\begin{array}{lll}F\left(\omega_{z}, \omega_{\varphi}\right) & \text { when } & \left|F\left(\omega_{z}, \omega_{\varphi}\right)\right| \geq A \\ 0 & \text { when } & \left|F\left(\omega_{z}, \omega_{\varphi}\right)\right|<A\end{array}\right.$

Where

$$
F\left(\omega_{z}, \omega_{\varphi}\right)=F F T(\Delta R(z, \varphi))
$$

$\Delta R(z, \varphi)$ is the wall deformation and $A$ is the threshold of the amplitude of the spectrum used to select the relative strong components which give major contributions to the wall perturbation. FFT represents the Fast Fourier Transformation. Then the smoothed wall surface can be calculated as

$$
\Delta R_{N}(z, \varphi)=F F T^{-1}\left(F_{N}\left(\omega_{z}, \omega_{\varphi}\right)\right)
$$

The spectrum of the wall profile of the launcher smoothed on the basis of the spectrum reconstruction method is plotted in Fig. 7, where the threshold $A=0.1$ was used in the synthesis procedure. The small amplitude components in the spectrum have been cancelled as shown in Fig. 7. One also can see that the high-frequency components have disappeared in the spectrum and that there are fewer frequency components in the spectrum shown in Fig. 7 compared to those plotted in Figs. 5 and 3. The wall profile of this mirrorline launcher is shown in Fig. 8, the minimum curvature radius is $29 \mathrm{~mm}$ in azimuthal direction, which is just 3.5 $\mathrm{mm}$ smaller than the launcher radius. The minimum curvature in longitudinal direction is $107.8 \mathrm{~mm}$. The wall profile shown in Fig. 8 is much smoother than that shown in Fig. 1 and the wall profile smoothed by lowpass filtering (Fig. 6)

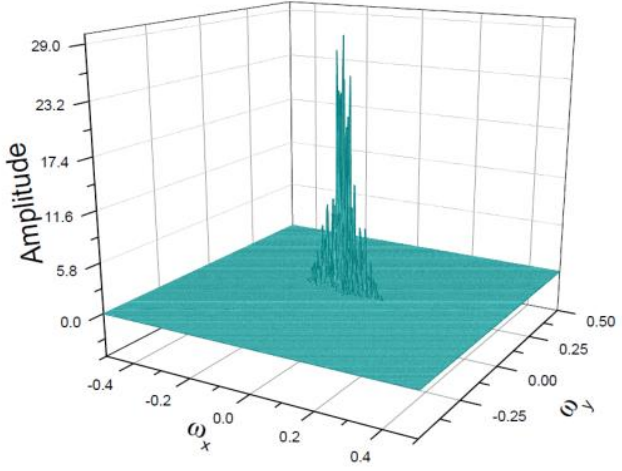

Fig. 7 Spectrum of the wall profile of the mirror-line launcher smoothed in terms of the spectrum reconstruction method. 


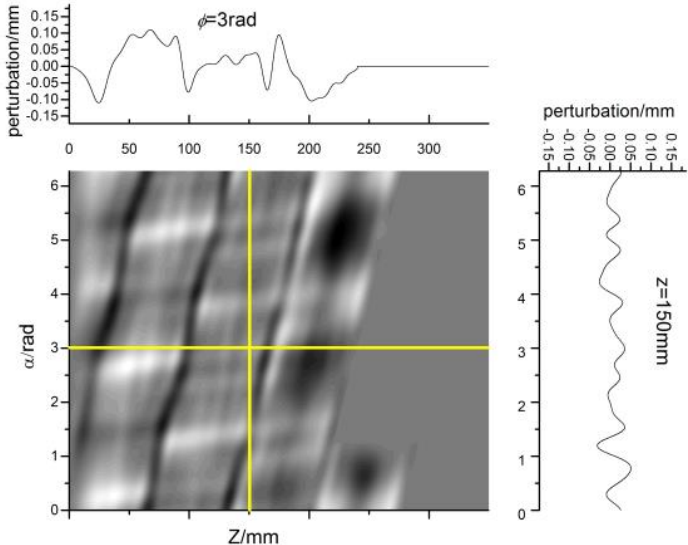

Fig. 8. Wall profile of the mirror-line launcher smoothed in terms of the spectrum reconstruction method.

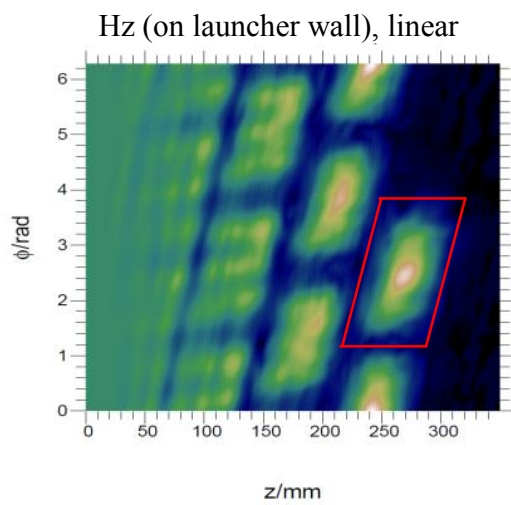

Fig. 9. $\mathrm{H}_{\mathrm{z}}$-field distribution on the wall of the mirror-line launcher smoothed in terms of the spectrum reconstruction method (linear scale, $0 \sim 1$ ).

The field distribution on the improved launcher wall is shown in Fig. 9. The Gaussian mode content of the RF beam at the launcher aperture is estimated as $96.1 \%$ according to the simulation result. High power measurements shows that the Gaussian mode content at the output window is $96 \%$, and the stray radiation was estimated by measurements to be $4 \%$ [9].

\section{Summary and Outlook}

Based on the spectrum reconstruction approach, a method for smoothing the launcher wall of numerically optimized high-power gyrotron quasi-optical output couplers has been developed at KIT. The curvature radii on the perturbed launcher wall have been remarkably increased. The improved launcher still provides an RF beam with high Gaussian mode content. The sensitivity of the improved launcher to fabrication errors and to frequency shifts will be investigated [8].

This work, supported by the European Communities under the contract of association between EURATOM and KIT, was carried out within the framework of the European Fusion Development Agreement. Part of this work was supported by Fusion for Energy under Grants F4E-2009-GRT-049 and within the European GYrotron Consortium (EGYC). The views expressed in this publication do not necessarily reflect the views of Fusion for Energy.

\section{References}

1. S.N. Vlasov and I.M. Orlova, Radiophysics \& Quantum Electronics, Vol. 17, pp. 115-119 (1974).

2. S.N. Vlasov, et al., Radio Eng. \& Electron Physics, Vol. 20, pp. 14-17'(1975).

3. G. G. Denisov, et al., Zapevalov, Int. J. Electron., Vol. 72, pp. 1079-1091 (1992).

4. A.V. Chirkov, et al., Radiophysics \& Quantum Electronics, Vol. 49, No. 5, pp. 344-353 (2006).

5. J. Jin, et al., IEEE Trans. Microw. Theory Techn., Vol. 57, No. 7, pp. 1661-1668 (2009).

6. D. I. Sobolev and G. G. Denisov, IEEE Trans. Plasma Science, Vol. 38, No. 10, pp. 2825-2830 (2010).

7. J. Jin, et al., IEEE Trans. Microw. Theory Techn., Vol. 65, No. 3, pp. 699-706 (2017).

8. J. Jin, et al., "A method to improve the tolerance of quasi-optical launchers for high power gyrotrons," to be submitted to IEEE Trans. Plasma Science.

9. T. Rzesnicki, et al., "2 MW Coaxial-Cavity PrePrototype Gyrotron for ITER - recent experiments with the modified gyrotron setup -", Proc. IRMMWTHz 2012, Wollongong, Australia, DOI: 10.1109/ IRMMW-THz.2012.6380413. 